\title{
Effects of multi orbital contributions in the angular-dependent ionization of molecules in intense few-cycle laser pulses
}

\author{
P. von den Hoff $\cdot$ I. Znakovskaya $\cdot$ S. Zherebtsov • \\ M.F. Kling • R. de Vivie-Riedle
}

Received: 26 August 2009 / Revised version: 11 November 2009 / Published online: 18 December 2009

(C) The Author(s) 2009. This article is published with open access at Springerlink.com

\begin{abstract}
We outline the details of our new method to calculate angular-dependent ionization probabilities based on electronic structure theory for diatomic and larger systems. To demonstrate its abilities, we compare our calculations to measured ionization probabilities of the four molecules $\mathrm{D}_{2}, \mathrm{~N}_{2}, \mathrm{O}_{2}$ and $\mathrm{CO}$ in the strong-field regime. The calculated angular distributions yield better agreement with the experimental data than those obtained from the widely used MO-ADK theory. For $\mathrm{CO}$ the measured angular distributions of ionic fragments indicate contributions to the ionization from both the HOMO and the HOMO-1 orbital, an effect that is addressed by the theory.
\end{abstract}

Keywords Angular resolved ionization - Strong laser fields · Electron flux · Molecules · Electronic structure theory $\cdot$ Velocity-map imaging $\cdot$ Few-cycle pulses

\section{Introduction}

Intense laser pulses of a few femtoseconds or even attoseconds are discussed as forthcoming tool for the coherent control of quantum dynamics in chemical systems. One perspective is to control molecular processes via the much faster electrons by preparing and directing an electronic

P. von den Hoff · R. de Vivie-Riedle $(\bowtie)$

Department für Chemie und Biochemie,

Ludwig-Maximilians-Universität München, 81377 München,

Germany

e-mail: regina.de_vivie@cup.uni-muenchen.de

I. Znakovskaya $\cdot$ S. Zherebtsov $\cdot$ M.F. Kling

Max-Planck Institute of Quantum Optics,

Hans-Kopfermann-Str. 1, 85748 Garching, Germany wavepacket to form or break bonds with a high selectivity. State of the art experiments on molecules in strong laser fields typically include ionization and subsequent processes like recollision excitation, high-harmonic generation, double ionization and dissociation. Here, we focus on the first step, the ionization caused by an electric field, which is a fundamental step for a multitude of phenomena observed in high-field physics.

Together with new advanced imaging techniques, which provide full momentum resolution of ionic fragments and electrons produced in a strong-field experiment, such as velocity-map imaging (VMI) or cold target recoil ion momentum spectroscopy (COLTRIMS), the knowledge of the angular-dependent ionization rates is particular important to understand or even predict the outcome of strong-field experiments. Therefore it is desirable to have a reliable method to calculate these angular dependencies.

The ionization of atoms in intense laser fields has been investigated extensively in the last decades, both theoretically and experimentally [1-7]. Common methods to calculate the ionization rates for atoms are the ADK (AmmosovDelone-Krainov) [3] and the strong-field approximation (SFA) [8]. Extensions for both models to the molecular case introducing molecular orbitals (MO) lead to the so called MO-SFA [9] and MO-ADK [10] models. Both approaches are proven to deliver good angular-dependent ionization rates for small systems in particular for linear molecules. Ionization from a single MO, essentially the highest occupied molecular orbital (HOMO) is assumed in both models. Recent strong-field experiments, however, give evidence that molecules with energetically close lying occupied valence orbitals allow ionization not only from the HOMO but also from the next lower lying orbitals, e.g. the HOMO-1 [11-13]. 
As research interest aims to investigate and control larger systems of especially biological interest, it is important to have a method that is capable to calculate accurate angulardependent ionization rates for larger molecules. Such molecules are not necessarily symmetric, so that the one-center approximation is not longer valid. Moreover the outer molecular orbitals exhibit smaller energetic spacings such that more orbitals may contribute to the angular-dependent ionization probabilities.

In this paper we outline the details of our new approach to calculate angular-dependent ionization probabilities based on electronic structure calculations that is capable to handle also complex molecular systems. In addition this ansatz is able to treat the ionization from more than one single MO. To demonstrate the ability of this new method, we compare our theoretical results with MO-ADK calculations and with experimental angular-dependent ionization probabilities. As test candidates we used the theoretically as well as experimentally well studied diatomic molecules $\mathrm{D}_{2}, \mathrm{~N}_{2}, \mathrm{O}_{2}$ and $\mathrm{CO}$. We selected the same pulse parameters as in the experiment, where we ionize the molecules with an intense fewcycle linearly polarized laser and detect the emitted ionic fragments $\left(\mathrm{D}^{+}, \mathrm{N}^{+}, \mathrm{O}^{+}\right.$and $\left.\mathrm{C}^{+}\right)$with full momentum resolution via VMI.

\section{Experimental setup}

The laser and vacuum setup used for the experiments was described in detail elsewhere [14]. For the measurements of the angular-dependent ionization of the molecules $\mathrm{D}_{2}$ and $\mathrm{CO}$ we generated $5 \mathrm{fs}$ and $4 \mathrm{fs}$, linearly polarized (LP) pulses at $760 \mathrm{~nm}\left(\mathrm{D}_{2}\right)$ and $740 \mathrm{~nm}(\mathrm{CO})$ and at intensities of 1.2 and $0.8 \times 10^{14} \mathrm{~W} / \mathrm{cm}^{2}$, respectively, with $3 \mathrm{kHz}$ repetition rate. For the $\mathrm{N}_{2}$ and $\mathrm{O}_{2}$ experiments, we used a slightly different laser setup generating $5 \mathrm{fs}$, linearly polarized pulses at $730 \mathrm{~nm}$ and an intensity of $1.6 \times 10^{14} \mathrm{~W} / \mathrm{cm}^{2}$ with $1 \mathrm{kHz}$ repetition rate. These few-cycle pulses were focused into the center of the ion optics of a VMI spectrometer using a spherical mirror. Ions and electrons that were generated at the crossing point of the laser ( $x$-axis) and an effusive molecular beam were accelerated and focused (along the $z$ axis) with the ion optics onto an MCP-phosphor screen assembly. The polarization of the laser was chosen along the $y$-axis, i.e. parallel to the $x y$-plane of the MCP detector. Images were recorded with a cooled CCD camera. Inversion of the recorded images using an iterative inversion procedure [15] allowed reconstruction of the original 3D ion momentum distributions.

\section{Experimental results}

In Fig. 1 cuts through the 3D momentum distributions of the ionic fragments $\left(\mathrm{D}^{+}\right.$from $\mathrm{D}_{2}(\mathrm{a}), \mathrm{N}^{+}$from $\mathrm{N}_{2}(\mathrm{~b})$,
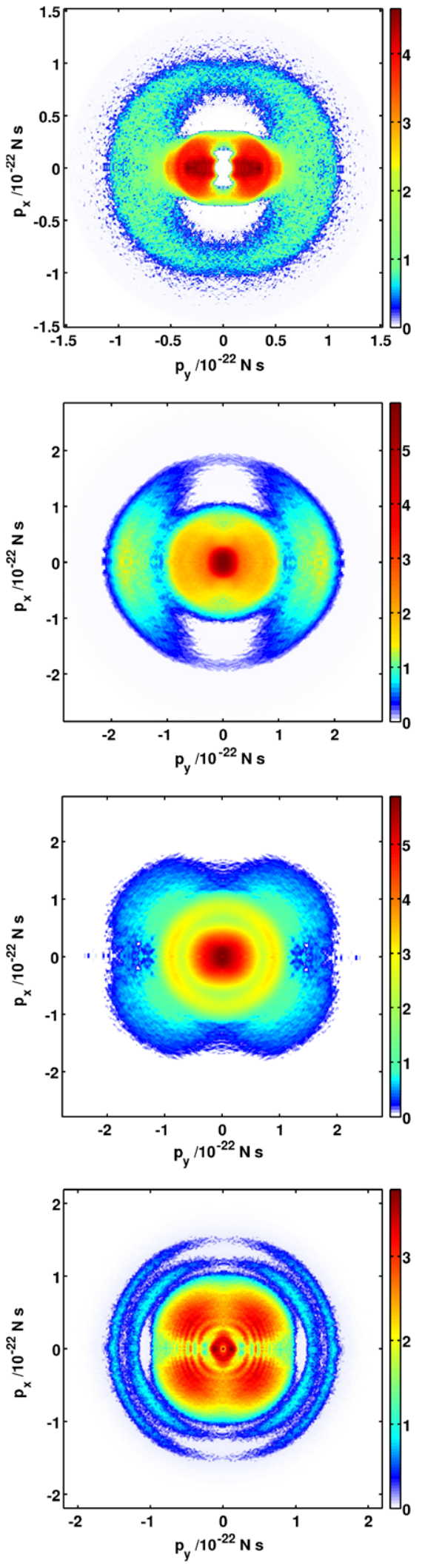

Fig. 1 Inverted two-dimensional momentum image (the laser polarization is horizontal) for the ionic fragments $\mathrm{D}^{+}$from $\mathrm{D}_{2}$ [16] (a) $\mathrm{N}^{+}$ from $\mathrm{N}_{2}(\mathbf{b}) \mathrm{O}^{+}$from $\mathrm{O}_{2}$ (c) $\mathrm{C}^{+}$from $\mathrm{CO}$ [12] (d) under the experimental conditions described in the text 
$\mathrm{O}^{+}$from $\mathrm{O}_{2}(\mathrm{c}), \mathrm{C}^{+}$from $\left.\mathrm{CO}(\mathrm{d})\right)$ that are created under the described experimental conditions in the $x y$-plane for $p_{z}=0$ are shown. The two-dimensional $\mathrm{D}^{+}$momentum distribution from the dissociative ionization of $\mathrm{D}_{2}$ (Fig. 1(a)) shows two main contributions [16]. The first ranges from $0-0.6 \times 10^{-22} \mathrm{Ns}$ and originates from bond softening and enhanced ionization [16]. The angular distributions in this momentum range show a clear maximum along and a distinct minimum perpendicular to the polarization axis. The second contribution ranges from $0.6-1.0 \times 10^{-22}$ Ns arising from recollision excitation [16] and exhibits also a maximum along the polarization axis and a weaker minimum perpendicular to this axis.

The two-dimensional $\mathrm{N}^{+}$momentum distribution from the dissociative ionization of $\mathrm{N}_{2}$ (Fig. 1(b)) shows also two main contributions. The first contribution ranges from 0 $1.2 \times 10^{-22} \mathrm{Ns}$ and exhibits background from the doubly ionized parent ion $\mathrm{N}_{2}^{2+}$, having the same mass to charge ratio and thus time-of-flight as $\mathrm{N}^{+}$and cannot be separated in the experimental detection. Thus the measured angular distribution at low momenta is a convolution of the distributions arising from the $\mathrm{N}^{+}$and $\mathrm{N}_{2}^{2+}$ ions and does not display the angular-dependent ionization rate. The second contribution ranges from $1.2-2.2 \times 10^{-22} \mathrm{Ns}$ and shows in agreement with the literature $[17,18]$ a maximum of the ionization rate along the polarization axis of the laser field and a minimum perpendicular to this axis. These ions partly arise from recollisional excitation of higher lying excited states in $\mathrm{N}_{2}^{+}$. Note that the recollisional excitation is not expected to significantly change the angular distributions.

In the two-dimensional $\mathrm{O}^{+}$momentum distribution from the dissociative ionization of $\mathrm{O}_{2}$ (Fig. 1(c)) the contribution below $1.2 \times 10^{-22}$ Ns comprise, like the low momentum distribution of $\mathrm{N}^{+}$(Fig. 1(b)), background from the doubly ionized parent ion $\mathrm{O}_{2}^{2+}$. The contribution between 1.2-2.2 $\times$ $10^{-22} \mathrm{Ns}$ exhibits maxima around $45^{\circ}, 135^{\circ}, 225^{\circ}, 315^{\circ}$ and minima around $0^{\circ}, 90^{\circ}, 180^{\circ}$ and $270^{\circ}$ and is as well as the angular-dependent ionization rate of $\mathrm{O}^{+}$in good agreement with the literature [17]. As in $\mathrm{N}_{2}$, these ions partly arise from recollisional excitation of higher lying excited states in $\mathrm{O}_{2}^{+}$.

In Fig. 1(d) the momentum distribution for the $\mathrm{C}^{+}$fragments from the dissociative ionization of $\mathrm{CO}$ is shown. Three circular contributions are visible in the ranges 0.3$0.9 \times 10^{-22} \mathrm{Ns}, 1.1-1.3 \times 10^{-22} \mathrm{Ns}$ and $1.4-1.6 \times 10^{-22} \mathrm{Ns}$. The first ring is the broadest and most intense. All three rings show similar angular distributions with minima around $0^{\circ}, 90^{\circ}, 180^{\circ}$ and $270^{\circ}$, however, with varying modulation depths. This angular distribution of the $\mathrm{C}^{+}$fragments differs from the findings reported by Alnaser et al. [19] and indicates contributions not only from the HOMO $(5 \sigma)$ but also from the HOMO-1 $\left(1 \pi_{x / y}\right)$ as will be discussed in this paper.

\section{Theoretical description}

The ionization probability of a molecule in a laser field is determined by the induced electron flux through the barrier of the combined molecular field and external electric field [4] (atomic units $\mathrm{m}=\hbar=\mathrm{e}=1$ are used throughout the paper):

$$
\begin{aligned}
& W(t)=\int_{S} j(r, t) \mathrm{d} S, \\
& \quad j(r, t)=-\frac{\mathrm{i}}{2}\left(\Psi(r, t) \nabla \Psi(r, t)^{*}-\Psi(r, t)^{*} \nabla \Psi(r, t)\right) .
\end{aligned}
$$

Here $j(r, t)$ is the electron flux density and $\Psi(r, t)$ the electronic wavefunction in the presence of the electric field inducing the electron flux $W(t)$. For the surface $S$ it is convenient to choose a plane perpendicular to the direction of the electric field. As the ionization takes place only at the edges of the electronic wavefunction, we choose, in the spirit of MO-ADK [10], the surface at the outer turning points of the electronic wavefunction to calculate the induced electron flux. Beyond these points the exponentially decaying term in the electron wavefunction becomes dominant. Here the wavefunction enters the classical forbidden region from where tunneling solely can occur.

In order to evaluate (1) the electronic wavefunction $\Psi(r)$ in the presence of the electric field is needed. In principle the electronic wavefunction can be calculated with the quantum chemistry package MOLPRO [20], but as this wavefunction is completely real the flux density $j(r, t)(1)$ is zero.

To overcome this problem, we calculate the electron flux by means of the electron density $\rho(r, t)$. Using the divergence theorem

$\int_{S} j(r, t) \mathrm{d} S=\int_{V^{\prime}} \nabla j(r, t) \mathrm{d} V^{\prime}$

and the continuity equation

$\frac{\mathrm{d}}{\mathrm{d} t} \rho(r, t)=-\nabla j(r, t)$

we rewrite (1) in the spirit of [21] in to

$W(t)=-\int_{V^{\prime}} \nabla j(r, t) \mathrm{d} V^{\prime}=\frac{\mathrm{d}}{\mathrm{d} t} \int_{V^{\prime}} \rho(r, t) \mathrm{d} V^{\prime}$.

In this case $V^{\prime}$ is the part of the total volume $V$ in which $\Psi(r)$ is defined that is spanned by the surface $S$ and the vector of the electric field (perpendicular to $S$ ) in the direction away from the nuclei. In order to calculate the tunneling probability $T(t ; S)$ for the electron passing through the potential barrier (e.g. the fractional amount of electrons passing through the surface $S$ into the volume $V^{\prime}$ ) we need the electronic wavefunction with and without the external field. In this sense we now integrate (4) from the initial time $t_{i}$ (corresponding to the electron wavefunction without the 
electric field) to the final time $t_{f}$ (corresponding to the electron wavefunction in the presence the electric field),

$T(t ; S)=\int_{V^{\prime}} \rho\left(r, t_{f}\right) \mathrm{d} V^{\prime}-\int_{V^{\prime}} \rho\left(r, t_{i}\right) \mathrm{d} V^{\prime}$.

In this way we perform quantum chemical calculation for a given molecule with and without a static electric field. The obtained electronic wavefunction is represented on a three-dimensional grid. The grid parameters where chosen to ensure that the electron density of both wavefunctions is below a threshold of $1.0 \mathrm{e}^{-6}$. Based on these two wavefunctions, we extract numerically the tunneling probability $T(t ; S)$ through the chosen surface $S$ according to (5). We then analyze $T(t ; S)$ induced by the electric field in the individual molecular orbitals to evaluate the ionization probability from the frontier orbitals, e.g. the HOMO. To calculate the angular-dependent ionization probability for a given electric field, we performed these calculations under various orientation angles with respect to the polarization of the applied external field. Within this procedure we follow the principal concept of [4] and MO-ADK [10], which are well established, but without restrictions to the one-center approximation and an effective core potential fixed at the center of mass. Moreover the calculated electronic wave functions contain the full electron correlation provided by the used quantum chemical method.

To treat ionization from more than one single orbital we solve the working equations derived above for a linear combination of the selected molecular orbitals. This implies a basis transformation rewriting the two orbitals (e.g. HOMO and HOMO-1) in the Slater determinant into the orbitals HOMO+HOMO-1 and HOMO-HOMO-1, allowing a coherent ionization of the electron from both initially chosen orbitals.

For the quantum chemical calculations it is particular important to check whether the energetic ordering of the molecular orbitals is correct and in agreement with experimental data. A wrong ordering will lead to a false prediction for the ionization probability. This problem arises in the calculations concerning the $\mathrm{N}_{2}$ molecule [22] and will be discussed later. For the MCSCF orbitals we used the canonical representation of the individual molecular orbitals as only for these orbitals the energy values are available. In the natural representation this is not the case, because the orbital energies provided by the quantum chemistry package MOLPRO are taken as the diagonal elements of the Fock-matrix in the basis of the natural orbitals. If the off diagonal elements in such a Fock-matrix are large the energetic order of the molecular orbitals can change. In the canonical representation the Fock-matrix is by definition diagonal and the energetic ordering of the molecular orbitals can be directly read out from the matrix.
Table 1 The ionization energy, equilibrium distance, and the expansion coefficients for the spherical harmonics $\mathrm{C}_{l}$ for $\mathrm{D}_{2}, \mathrm{~N}_{2}, \mathrm{O}_{2}$ and $\mathrm{CO}$

\begin{tabular}{lllllll}
\hline & $\mathrm{I}_{p}$ & $\mathrm{R} \AA$ & $\mathrm{C}_{l=0}$ & $\mathrm{C}_{l=2}$ & $\mathrm{C}_{l=4}$ & \\
\hline $\mathrm{D}_{2}\left(\sigma_{g}\right)$ & 15.47 & 0.742 & 2.51 & 0.06 & 0.00 & \\
$\mathrm{~N}_{2}\left(\sigma_{g}\right)$ & 15.58 & 1.098 & 2.02 & 0.78 & 0.04 & \\
$\mathrm{O}_{2}\left(\pi_{g}\right)$ & 12.03 & 1.208 & 0.00 & 0.62 & 0.03 & \\
\hline & & & $\mathrm{C}_{l=0}$ & $\mathrm{C}_{l=1}$ & $\mathrm{C}_{l=2}$ & $\mathrm{C}_{l=3}$ \\
\hline $\mathrm{CO}(\sigma)$ & 14.01 & 1.128 & 1.43 & 0.76 & 0.28 & 0.02 \\
\hline
\end{tabular}

For the MO-ADK calculations used as reference for the above introduced method (see Sect. 5), we follow the procedure described in [10]. The required expansion coefficients for the spherical harmonics $C_{l}$ (see [10]) as well as the ionization energies and equilibrium distances are also taken from [10] and listed in Table 1.

For both approaches we assume a static electric field during the ionization corresponding to the maximum intensity of the experimental ultra-short (4 and $5 \mathrm{fs}$ ) light pulse. This is justified as the other half cycles are less intense and thus have a significantly lower ionization probability.

\section{Results and discussion}

We used the above introduced method to calculate the angular-dependent ionization probabilities for the molecules $\mathrm{D}_{2}, \mathrm{~N}_{2}, \mathrm{O}_{2}$ and $\mathrm{CO}$ and compare the results with the angular-dependent ionization probabilities calculated with MO-ADK theory as well as with experimentally measured angular-dependent ionization rates. Note that in the experiments an ensemble of molecules is ionized that is not aligned or oriented. The angular distribution of detected ionic fragments reflects the angular-dependent ionization rates for laser pulses with sufficiently short duration and low intensity [23]. Moreover no significant change of the angular distributions is expected neither through recollisional excitation nor through dynamic or post ionization alignment [23]. For all presented examples the electronic wavefunction with and without the presence of the external electric field is calculated with the quantum chemistry package MOLPRO [20] applying the default criteria for convergence. We used sufficiently large basis sets including polarization and diffuse functions in all cases except for $\mathrm{N}_{2}$ (see Sect. 5.2). Both types of functions improve the description of the electron at large distances from the nuclei. In case of CASSCF calculations all electrons except the core electrons are included in the active space. In order to be able to compare the individual ionization probabilities from the experiment and from the theoretical calculations we have normalized all data, setting the largest value to one. 


\subsection{Angular-dependent ionization probabilities for $\mathrm{D}_{2}$}

The $\mathrm{D}_{2}$ calculations were performed on the $\operatorname{CASSCF}(2,8) /$ $6-311++\mathrm{G}^{* *}$ level of theory and for an electric field strength of 0.058 a.u. (corresponding to the intensity of $1.2 \times 10^{14} \mathrm{~W} / \mathrm{cm}^{2}$ used in the experiment). To scan the angular dependence, we performed the quantum chemistry calculation for 37 orientation of the molecule with respect to the applied electric field ranging from $0^{\circ}$ (molecular axis parallel to the laser polarization) to $360^{\circ}$ with a step size of $10^{\circ}$. The resulting angular-dependent ionization probability is plotted in Fig. 2 as black solid line. The black crosses indicate the actually calculated angles. Our calculations show a clear maximum in the ionization probability for molecular orientations along the laser polarization axis and a week minimum for orientations perpendicular to this axis. We find that the ionization is 2.1 times higher for parallel alignment $\left(0^{\circ}\right)$ than for perpendicular alignment $\left(90^{\circ}\right)$.

The experimental angular-dependent ionization rate is derived from the VMI momentum image (Fig. 1(a)) by integration over the contribution arising from recollision excitation $\left(0.6-1.0 \times 10^{-22} \mathrm{Ns}\right)$ and is plotted in Fig. 2 as gray solid line. The angular dependence exhibits a maximum along the polarization axis and a minimum perpendicular to this axis. The parallel vs. perpendicular ratio is around 3.0.

The results of the angle-dependent MO-ADK calculations for the same field strength of 0.058 a.u. are plotted as black dashed line in Fig. 2 and show roughly the same ionization probability for all angles. The parallel vs. perpendicular ratio calculated according to MO-ADK is 1.2.

From Fig. 2 it can be seen that our new approach is in good agreement with the experimental data. Not only the parallel vs. perpendicular ratio is reproduced well but also

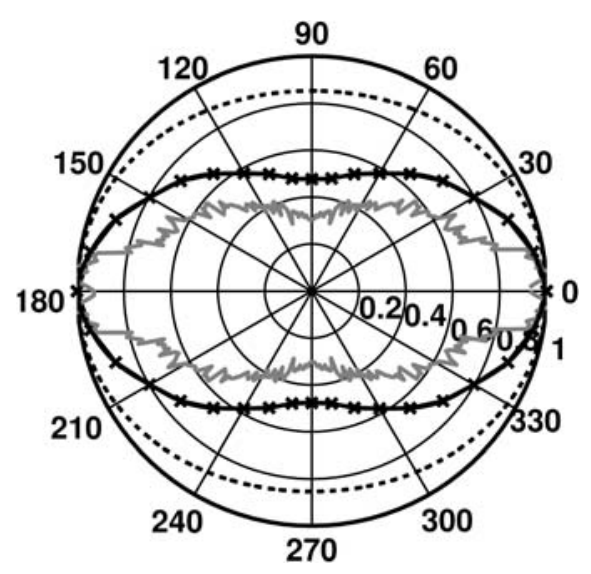

Fig. 2 Black solid line: calculated angular-dependent ionization probability for the HOMO of $\mathrm{D}_{2}$ (the crosses indicate the actually calculated points). Black dashed line: angular-dependent ionization probability for the HOMO of $\mathrm{D}_{2}$ calculated with the MO-ADK model. Gray solid line: experimentally measured angular-dependent ionization rate for $\mathrm{D}_{2}$ the overall shape of the angular distribution. The MO-ADK calculations predict a lower parallel vs. perpendicular ratio as well as a false shape of the total angular distribution. Presumably this is due to the one-center approximation. To describe the $\sigma_{g}$ orbital of the $\mathrm{D}_{2}$ molecule a huge contribution of the spherical harmonic with $l=0$ is used in the linear combination and only a week contribution of $l=2(0.06$ see Table 1) to break the spherical symmetry. Thus the shape of the $\sigma_{g}$ orbital is not reproduced correctly. Moreover the potential formed by the nuclei and one of the two electrons is treated with an effective potential $\left(-\frac{Z_{\text {eff }}}{r}\right)$ which presumably also contributes to the error made for perpendicular orientations. Here the tunneling electron is affected by both nuclei. In our method all these effects are already included in the quantum chemistry calculations.

\subsection{Angular-dependent ionization probabilities for $\mathrm{N}_{2}$}

The electric field strength was set to 0.067 a.u., equivalent to the experimentally used intensity of $1.6 \times 10^{14} \mathrm{~W} / \mathrm{cm}^{2}$. In the case of $\mathrm{N}_{2}$ Hartree Fock and MCSCF calculations carried out with basis sets larger than STO-3G predict the wrong energetic ordering of the valence orbitals. The HOMO in the $\mathrm{N}_{2}$ molecule is the $3 \sigma_{g}$ orbital, not the $1 \pi_{u}$. This is a well known problem in quantum chemistry [22]. The correct order leads to the experimentally found $\mathrm{N}_{2}^{+}$ ground state of ${ }^{2} \Sigma_{g}^{+}$symmetry. The interchanged ordering would not only result in the wrong ionic ground state of $\mathrm{N}_{2}^{+}$ $\left({ }^{2} \Pi_{g}\right)$ but also in the wrong angular-dependent ionization rates. Even molecular orbitals derived from MCSCF calculations including electron correlation also for spin paired electrons cannot overcome this problem. For $\mathrm{N}_{2}$ we therefore used the HF/STO-3G level of theory. In the isoelectronic molecule $\mathrm{CO}$, treated below, the orbital ordering is correct. Here the outer molecular orbitals $4 \sigma$ and the $5 \sigma$ have the same symmetry and can interact with each other. In $\mathrm{N}_{2}$ the corresponding orbitals $\left(2 \sigma_{u}\right.$ and $\left.3 \sigma_{g}\right)$ belong to different irreducible representations and cannot repel each other. In the HF/STO-3G calculations the $3 \sigma_{g}$ orbital is "accidentally right" and the highest occupied orbital. Therefore we refrain from the higher accuracy normally provided by larger basis sets in favor to maintain the right molecular orbital order. For the angular dependence we scan, as in the previous section, over 37 orientation of the molecule with respect to the applied electric field ranging from $0^{\circ}$ (molecular axis parallel to the laser polarization) to $360^{\circ}$ with a constant step size of $10^{\circ}$. The angular-dependent ionization probability is plotted in Fig. 3 as black solid line and shows a maximum in the ionization probability for molecules oriented along the laser polarization axis and a minimum for orientations perpendicular to this axis (parallel vs. perpendicular ratio is 3.5). This calculated angular distribution is in reasonable agreement with the experimental one, derived 


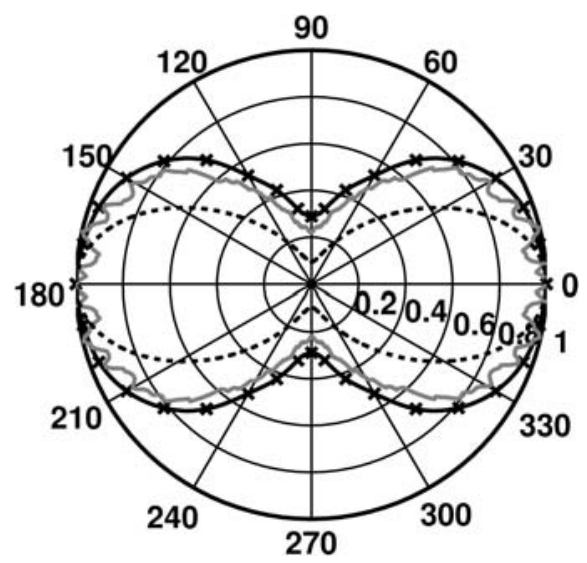

Fig. 3 Black solid line: calculated angular-dependent ionization probability for the HOMO of $\mathrm{N}_{2}$ (the crosses indicate the actually calculated points). Black dashed line: angular-dependent ionization probability for the HOMO of $\mathrm{N}_{2}$ calculated with the MO-ADK model. Gray solid line: experimentally measured angular-dependent ionization rate for $\mathrm{N}_{2}$

from the VMI spectrum (Fig. 1(b)) by integration over the momentum range from $1.1 \mathrm{eV}$ to $2.2 \times 10^{-22} \mathrm{Ns}$ and shown in Fig. 3 as gray solid line. The measured parallel vs. perpendicular ratio is around 4.5 which is in excellent agreement with the literature [18] (4.5).

The angular-dependent ionization probabilities calculated according to the MO-ADK theory (field strength of 0.067 a.u.) are plotted in Fig. 3 as black dashed line. Its shape shows also a reasonable agreement with the experimental data. The predicted parallel vs. perpendicular ratio, however, with 10.5 is far off from the experimental value.

\subsection{Angular-dependent ionization probabilities for $\mathrm{O}_{2}$}

The electronic wavefunctions for the $\mathrm{O}_{2}$ molecule are calculated on the CASSCF $(12,10) / 6-311+\mathrm{G}^{*}$ level of theory and an electric field strength of 0.067 a.u. equivalent to the experimentally used intensity. $\mathrm{O}_{2}$ in its electronic ground is a diradical where both of the degenerated $1 \pi^{*}$ orbitals $\left(\pi_{g}\right.$ symmetry) are occupied with one electron. The degeneration of the two orbitals is not lifted in the presence of the electronic fields and the ionization can occur from both orbitals. To accommodate this fact, we allow the electron a priori to tunnel from both orbitals with the same probability, by calculating the ionization probability from the linear combination of the $1 \pi_{x}^{*}$ and $1 \pi_{y}^{*}$ orbital. The resulting angular-dependent ionization pattern is shown in Fig. 4 as black solid line and fits very good with the experimental data (Fig. 4 gray solid line). The experimental data have been derived by integration of the VMI momentum image over the contribution between 1.1 and $2.2 \times 10^{-22} \mathrm{Ns}$ and is in good agreement with the literature [17].

For the MO-ADK calculations we used according to the literature $[10,17]$ only the $\pi_{g}$ orbital lying in the plane

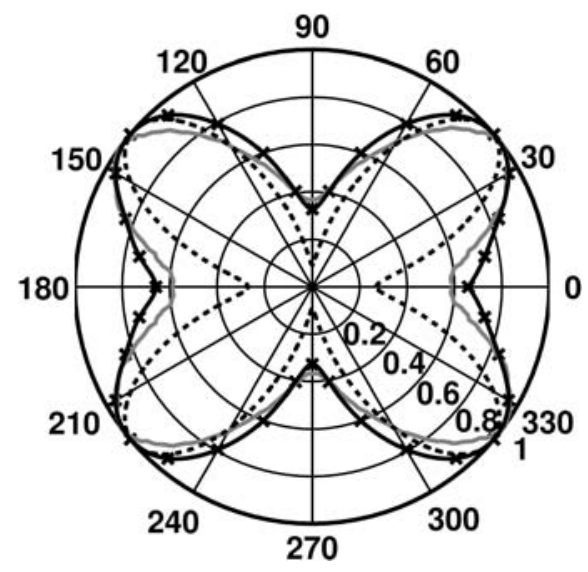

Fig. 4 Black solid line: calculated angular-dependent ionization probability for the HOMO (Linear combination of $1 \pi_{x}^{*}$ and $1 \pi_{y}^{*}$ ) of $\mathrm{O}_{2}$ (the crosses indicate the actually calculated points). Black dashed line: angular-dependent ionization probability for the $\mathrm{HOMO}$ of $\mathrm{O}_{2}$ calculated with the MO-ADK model. Gray solid line: experimentally measured angular-dependent ionization rate for $\mathrm{O}_{2}$

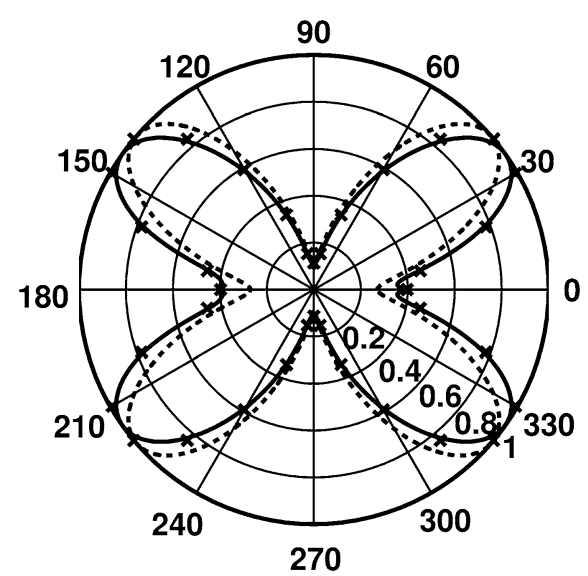

Fig. 5 Black solid line: calculated angular-dependent ionization probability for the HOMO of $\mathrm{O}_{2}$ (the crosses indicate the actually calculated points). Black dashed line: angular-dependent ionization probability for the $\mathrm{HOMO}$ of $\mathrm{O}_{2}$ calculated with the MO-ADK model

perpendicular to the rotation axis used for the angulardependent calculations. Again the field strength corresponds to the intensity used in the experiment. In general the results are also in good agreement with the experimental (Figs. 4 and 5 black dashed line) data apart from the fact, that the minimum in ionization rate for parallel and perpendicular alignment to the laser field is overestimated. According to our calculations this can be assigned to the assumption that the ionization takes place from one orbital only. This is verified in Fig. 5, when we likewise include only one $\pi_{g}$ in our new approach (Fig. 5 black solid line) we reproduce a similar result as the MO-ADK theory. Only when we include both $\pi_{g}\left(\pi_{x}\right.$ and $\left.\pi_{y}\right)$ orbitals the minima around $0^{\circ}, 90^{\circ}$, $180^{\circ}$ and $270^{\circ}$ and the overall shape fit much better with the experiment (see Fig. 4). 


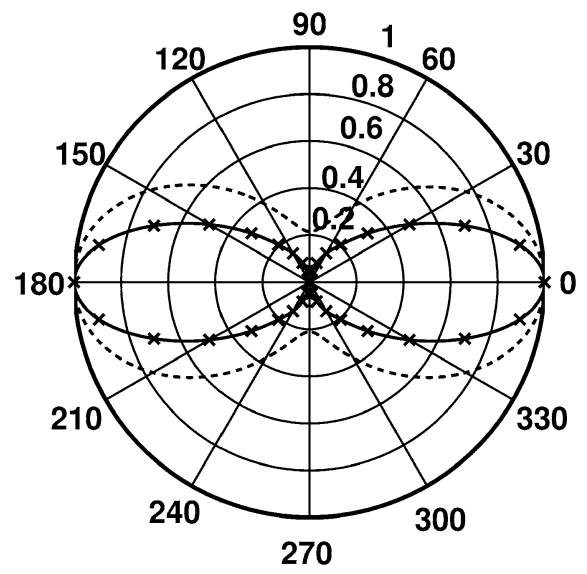

Fig. 6 Black solid line: calculated angular-dependent ionization probability for the HOMO $(5 \sigma)$ of $\mathrm{CO}$ (the crosses indicate the actually calculated points). Black dashed line: angular-dependent ionization probability for the $\mathrm{HOMO}$ of $\mathrm{CO}$ calculated with the MO-ADK model

\subsection{Angular-dependent ionization probabilities for $\mathrm{CO}$}

The CO molecule serves here as an example for heteronuclear diatomic molecules. For this class of molecules the molecular orbitals are not symmetrically distributed over the molecule, but partially localized on one of the nuclei. Thus one can expect different ionization probabilities for different orientation ( $\mathrm{CO}$ vs. $\mathrm{OC}$ ) of the molecule with respect to the laser polarization. This effect is averaged out without phase stabilization and for long laser pulses, while it creates an asymmetric angular distribution in an ensemble of the ionized molecules with phase stabilized pulses. In this case one can expect an asymmetry caused by the field induced ionization for the $\mathrm{CO}$ molecule. Indeed this effect (amongst others) has been studied experimentally and theoretically with the presented method in [12]. Here we will focus on the symmetric angular distributions produced with non phase stabilized laser pulses.

The electronic wavefunctions for the $\mathrm{CO}$ molecule are calculated on the CASSCF $(10,11) / 6-311+\mathrm{G}^{*}$ level of theory and an electric field strength of 0.047 a.u. equivalent to the experimental intensity. When we assume the ionization only from the HOMO $(5 \sigma)$ our calculated angulardependent ionization probabilities (Fig. 6 black solid line) matches reasonably well with the angular distribution calculated with MO-ADK (Fig. 6 black dashed line) and the experimental findings from Alnaser and coworkers [19] who studied the angular-dependent fragmentation pattern from $\mathrm{CO}^{2+}$.

However, this angular-dependent ionization probability does not reflect the angular distribution measured under the above described experimental conditions showing maxima around $45^{\circ}, 135^{\circ}, 225^{\circ}, 315^{\circ}$ and minima around $0^{\circ}, 90^{\circ}$, $180^{\circ}$ and $270^{\circ}$ (see Fig. 7 gray solid line). These angulardependent ionization rates are obtained by integration of the

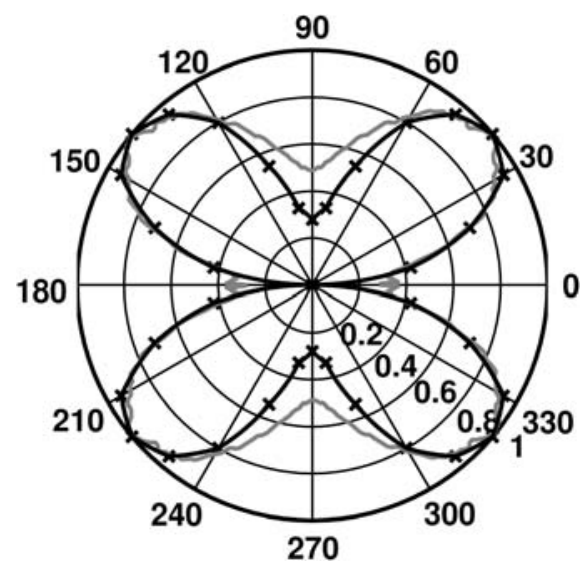

Fig. 7 Black solid line: calculated angular-dependent ionization probability for the HOMO and HOMO-1 $\left(5 \sigma\right.$ and $\left.1 \pi_{x / y}\right)$ of CO. Gray solid line: experimentally measured angular-dependent ionization rate for $\mathrm{CO}$

VMI momentum map over the most pronounced contribution between 0.3 and $0.9 \times 10^{-22} \mathrm{Ns}$. If we now include in our calculations the HOMO- $1\left(1 \pi_{x / y}\right)$, we obtain the angular distribution plotted in Fig. 7 (black solid line) which is in good agreement with the experimental data. Even the more distinct minima for parallel than for perpendicular alignment can be reproduced within our calculations.

\section{Conclusion}

We presented experimental and theoretical results on orbital symmetry effects on the recorded angular distributions of ions from the dissociative ionization of the molecules $\mathrm{D}_{2}$, $\mathrm{N}_{2}, \mathrm{O}_{2}$ and $\mathrm{CO}$ in intense few-cycle laser fields. Our theoretical approach goes beyond the one orbital and one-center approximation in MO-ADK. We compare the theoretical results based on the new approach with MO-ADK calculations and with experimental angular-dependent ionization probabilities. Very good agreement is found between the new theoretical and the experimental data. The angular-dependent ionization rate at intensities close to $10^{14} \mathrm{~W} / \mathrm{cm}^{2}$ for $\mathrm{D}_{2}$, $\mathrm{N}_{2}$ and $\mathrm{O}_{2}$ is found to reflect the orbital symmetries of the HOMO orbitals in agreement with earlier observations [19], for $\mathrm{CO}$, however, both the HOMO and the HOMO-1 orbitals contribute.

Acknowledgement We acknowledge F. Krausz and M.J.J. Vrakking for their support, fruitful discussions and the use of specialized equipment. We are grateful for support by the DFG via the Emmy-Noether program and the Cluster of Excellence: Munich Center for Advanced Photonics (www.munich-photonics.de).

Open Access This article is distributed under the terms of the Creative Commons Attribution Noncommercial License which permits any noncommercial use, distribution, and reproduction in any medium, provided the original author(s) and source are credited. 


\section{References}

1. A.M. Perelomov, V.S. Popov, M.V. Terent'ev, Sov. Phys. JETP 23, 924 (1966)

2. A.M. Perelomov, V.S. Popov, M.V. Terent'ev, Sov. Phys. JETP 23, 924 (1966)

3. M. Ammosov, N. Delone, V. Krainov, Sov. Phys. JETP 64, 1191 (1986)

4. B.M. Smirnov, M.I. Chibisov, Sov. Phys. JETP 22, 585 (1966)

5. B. Buerke, D.D. Meyerhofer, Phys. Rev. A 69, 051402 (2004)

6. S. Augst, D. Strickland, D.D. Meyerhofer, S.L. Chin, J.H. Eberly, Phys. Rev. Lett. 63, 2212 (1989)

7. S. Augst, A. Talebpour, S.L. Chin, Y. Beaudoin, M. Chaker, Phys. Rev. A 52, 914 (1995)

8. F.H.M. Faisal, A. Becker, J. Muth-Böhm, Laser Phys. 9, 115 (1999)

9. J. Muth-Böhm, A. Becker, F.H.M. Faisal, Phys. Rev. Lett. 85, $2280(2000)$

10. X.M. Tong, Z. Zhao, C.D. Lin, Phys. Rev. A 66, 033402 (2002)

11. B.K. McFarland, J.P. Farrell, P.H. Bucksbaum, M. Gühr, Science 322, 1232 (2008)

12. I. Znakovskaya, P. von den Hoff, S. Zherebtsov, A. Wirth, O. Herrwerth, M. Vrakking, R. de Vivie-Riedle, M. Kling, Phys. Rev. Lett. 103, 103002 (2009)

13. O. Smirnova, Y. Mairesse, S. Patchkovskii, N. Dudovich, D. Villeneuve, P. Corkum, M.Y. Ivanov, Nature 460, 972 (2009)

14. M. Schultze, E. Goulielmakis, M. Uiberacker, M. Hofstetter, J. Kim, D. Kim, F. Krausz, U. Kleineberg, New J. Phys. 9, 243 (2007)
15. M.J.J. Vrakking, Rev. Sci. Instrum. 72, 4084 (2001)

16. M.F. Kling, C. Siedschlag, A.J. Verhoef, J.I. Khan, M. Schultze, T. Uphues, Y. Ni, M. Uiberacker, M. Drescher, F. Krausz, M.J.J. Vrakking, Science 312, 246 (2006)

17. D. Pavičić, K.F. Lee, D.M. Rayner, P.B. Corkum, D.M. Villeneuve, Phys. Rev. Lett. 98, 243001 (2007)

18. I. Litvinyuk, K.F. Lee, P. Dooley, D. Rayner, D. Villeneuve, P.B. Corkum, Phys. Rev. Lett. 90, 233003 (2003)

19. A.S. Alnaser, C.M. Maharjan, X.M. Tong, B. Ulrich, P. Ranitovic, B. Shan, Z. Chang, C.D. Lin, C.L. Cocke, I.V. Litvinyuk, Phys. Rev. A 71, 031403 (2005)

20. H.-J. Werner, P.J. Knowles, R. Lindh, F.R. Manby, M. Schütz, P. Celani, T. Korona, A. Mitrushenkov, G. Rauhut, T.B. Adler, R.D. Amos, A. Bernhardsson, A. Berning, D.L. Cooper, M.J.O. Deegan, A.J. Dobbyn, F. Eckert, E. Goll, C. Hampel, G. Hetzer, T. Hrenar, G. Knizia, C. Köppl, Y. Liu, A.W. Lloyd, R.A. Mata, A.J. May, S.J. McNicholas, W. Meyer, M.E. Mura, A. Nicklass, P. Palmieri, K. Pflüger, R. Pitzer, M. Reiher, U. Schumann, H. Stoll, A.J. Stone, R. Tarroni, T. Thorsteinsson, M. Wang, A. Wolf, Molpro, version 2006.1 (2006)

21. I. Barth, H.-C. Hege, H. Ikeda, A. Kenfack, M. Koppitz, J. Manz, F. Marquardt, G.K. Paramonov, Chem. Phys. Lett. 481, 118 (2009)

22. A. Szabo, N.S. Ostlund, Modern Quantum Chemistry (Dover, New York, 1996)

23. X.M. Tong, Z.X. Zhao, A.S. Alnaser, S. Voss, C.L. Cocke, C.D. Lin, J. Phys. B: At. Mol. Opt. Phys. 38, 333 (2005) 